OPEN ACCESS

Edited by:

Kevin Mason,

Nationwide Children's Hospital,

United States

Reviewed by:

Timothy Murphy,

University at Buffalo, United States

Stephen Peter Kidd,

University of Adelaide, Australia

${ }^{*}$ Correspondence:

Kristian Riesbeck

kristian.riesbeck@med.lu.se

Specialty section: This article was submitted to

Bacteria and Host,

a section of the journal

Frontiers in Cellular and

Infection Microbiology

Received: 30 November 2021

Accepted: 19 January 2022

Published: 11 February 2022

Citation:

Janoušková M, Straw ML, Su Y-C and

Riesbeck K (2022) Gene Expression

Regulation in Airway Pathogens:

Importance for Otitis Media.

Front. Cell. Infect. Microbiol. 12:826018.

doi: 10.3389/fcimb.2022.826018

\section{Gene Expression Regulation in Airway Pathogens: Importance for Otitis Media}

\author{
Martina Janoušková, Megan Laura Straw, Yu-Ching Su and Kristian Riesbeck* \\ Clinical Microbiology, Department of Translational Medicine, Faculty of Medicine, Lund University, Malmö, Sweden
}

Otitis media (OM) is an inflammatory disorder in the middle ear. It is mainly caused by viruses or bacteria associated with the airways. Streptococcus pneumoniae, Haemophilus influenzae and Moraxella catarrhalis are the three main pathogens in infection-related OM, especially in younger children. In this review, we will focus upon the multifaceted gene regulation mechanisms that are well-orchestrated in $S$. pneumoniae, $H$. influenzae, and $M$. catarrhalis during the course of infection in the middle ear either in experimental $\mathrm{OM}$ or in clinical settings. The sophisticated findings from the past 10 years on how the othopathogens govern their virulence phenotypes for survival and host adaptation via phase variation- and quorum sensing-dependent gene regulation, will be systematically discussed. Comprehensive understanding of gene expression regulation mechanisms employed by pathogens during the onset of OM may provide new insights for the design of a new generation of antimicrobial agents in the fight against bacterial pathogens while combating the serious emergence of antimicrobial resistance.

Keywords: gene expression regulation, Haemophilus influenzae, Moraxella catarrhalis, otitis media, Streptococcus pneumoniae

\section{INTRODUCTION}

Otitis media $(\mathrm{OM})$ covers a spectrum of middle ear $(\mathrm{ME})$ inflammatory disorders that are caused by various irritating agents and pathogens. In low to middle-income countries, $\mathrm{OM}$ is the main medical condition for antibiotics prescription and surgeries, and deafness in children, respectively. Despite $\mathrm{OM}$ is a relatively mild condition that in many cases heals spontaneously, it can cause severe illness and thousands of OM-related deaths have been reported annually (Monasta et al., 2012; Schilder et al., 2016).

Streptococcus pneumoniae (Spn), non-typeable Haemophilus influenzae (NTHi), and Moraxella catarrhalis (Mcat) are the three main othopathogens isolated from OM patients (Biesbroek et al., 2014; Mills et al., 2015; Lee et al., 2020). The course of othopathogen-dependent OM involves bacterial colonization on the ME epithelium (Hendrixson and St Geme, 1998; Thornton et al., 2011). Bacterial infection in the ME usually occurs as a secondary infection to viral infection in the nasopharynx that subsequently progresses to ME via the Eustachian tube (Jossart et al., 1994; Jiang et al., 1999) (Figure 1). According to general guidelines in various countries, antimicrobial agents, depending on the age group of children are the treatment of choice of OM. An effective vaccine for 
bacterial-related OM is still unavailable (Jalalvand and Riesbeck, 2018). Child immunization programs including conjugated pneumococcal vaccines have, however, decreased the incidence of Spn-related OM in most countries (Littorin et al., 2016; Littorin et al., 2021).

To overcome the hostile environment in the human airway during OM, bacteria are forced to have sophisticated gene regulation expression machineries for optimum survival and adaptation especially in the nasopharynx and ME. In this review, we will mainly focus on the most studied gene regulation mechanisms of Spn, NTHi and Mcat that are associated with $\mathrm{OM}$ in the past decade.

\section{Streptococcus pneumoniae}

Streptococcus pneumoniae is a Gram-positive diplococcus with capsulated cell wall (Brooks and Mias, 2018). The bacteria asymptomatically colonize (carriage phase) the human upper airway as a part of commensal microbiota in a healthy individual. The carriage phase is essential for pneumococcal pathogenic transition to other sterile sites causing symptomatic infections, such as $\mathrm{OM}$ in the $\mathrm{ME}$, in individuals with an immature or weakened immune system (Chao et al., 2014; Coleman et al., 2018; Weiser et al., 2018).

The transition of Spn from nasopharyngeal colonization to ME infection is a multifactorial process. The pathogen needs to adapt to the diverse environmental conditions in the human airway during transition between niches. This includes the varying levels of nutritional status, acidities, oxygen, carbon dioxide, temperature, and a myriad of host antimicrobial factors (Kloosterman and Kuipers, 2011; Manzoor et al., 2015; Paixao et al., 2015a; Paixao et al., 2015b; Man et al., 2017; Aprianto et al., 2018). The establishment of Spn colonization

A
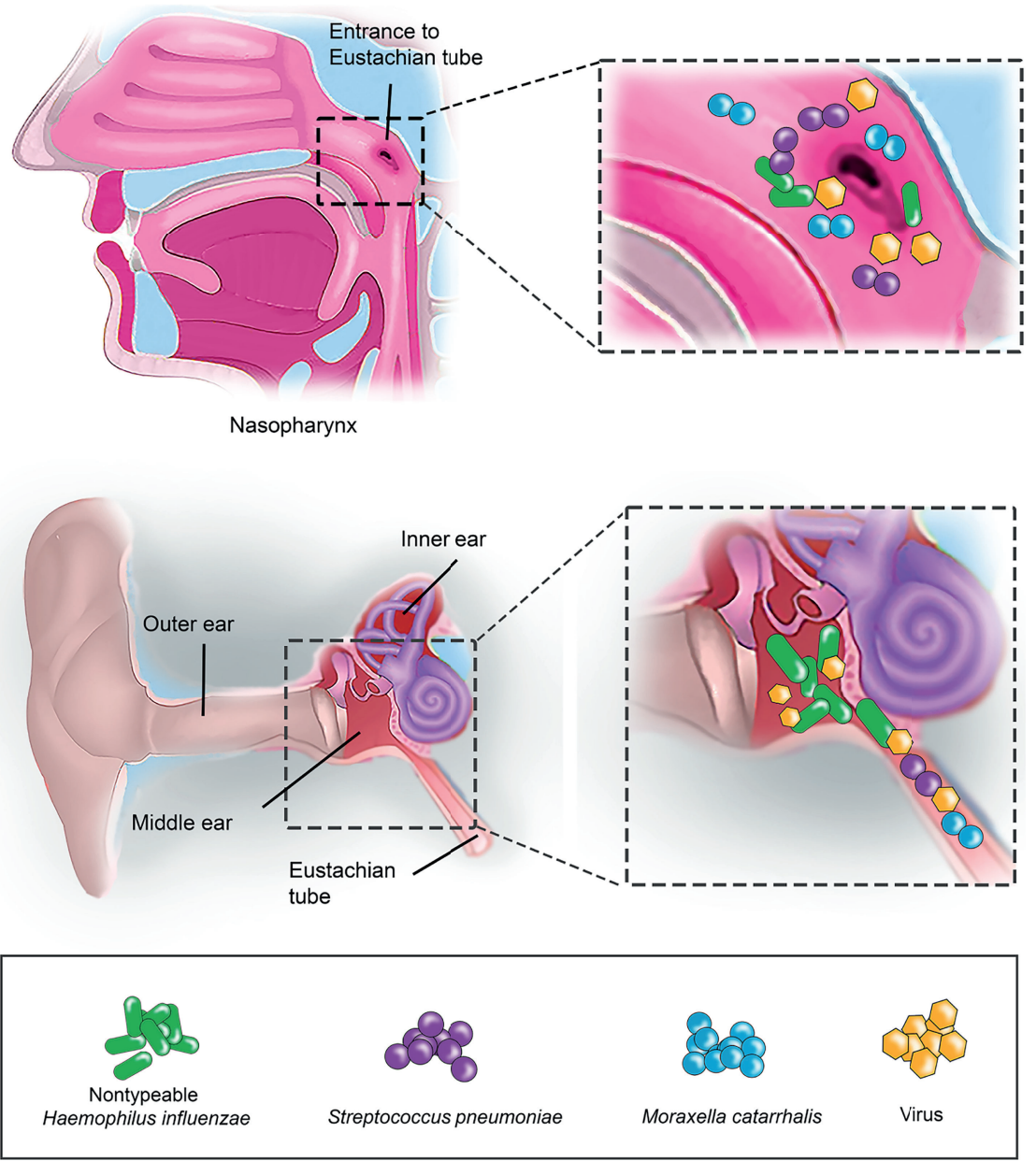

FIGURE 1 | Bacterial colonisation in human nasopharynx and middle ear. (A) Nasopharynx is the primary entrance for airway pathogens. Small insert (left panel) indicates the enlarged view of the entrance to Eustachian tube in nasopharynx. Airway pathogens such as nontypeable $H$. influenzae (NTHi) (indicated as green rod), S. pneumoniae (Spn) (purple sphere) and M. catarrhalis (Mcat) (blue sphere) that initially colonize the nasopharynx as part of the commensal microbiota can, however, migrate into the middle ear (ME) via the Eustachian tube. (B) Co-infection of airway pathogens and viruses in the ME. Airway pathogens (NTHi, Spn and Mcat) that have successfully entered the Eustachian tube can travel into the ME and colonize as a biofilm. This results in a middle ear inflammation triggered by the host immune response, and subsequently leads to the onset of OM. Virus (yellow hexagon) infection is often preceding the bacterial infection in OM. 
in the nasopharynx and ME requires fine-tuned gene expression of a plethora of virulence and metabolism factors in response to the environmental conditions at target niches. Pneumococcal virulence factors, as recently reviewed in detail by Brooks et al. and Weiser et al., promote biofilm formation and contribute to pneumococcal adherence to airway epithelial cells, evasion of mucosal clearance by the host immune system, and to outcompete co-colonizing bacteria or resident flora (Brooks and Mias, 2018; Weiser et al., 2018).

\section{Gene Expression During Colonization}

Besides the host immune factors, virus infection, the cocolonizing microbiome, and exposure to inhalable particle matters at the nasopharynx and ME can also promote Spn infection in the ME. Host norepinephrine, extracytoplasmic $\mathrm{ATP}$, and nutrients (i.e., $\mathrm{N}$-acetylneuraminic acid and $\mathrm{N}$ acetylglucosamine) released from the damaged nasopharyngeal tissue following a simultaneous viral infection promote pneumococcal dispersal from nasopharyngeal biofilm colonization and migration to the ME (Marks et al., 2013; Chao et al., 2014; Paixao et al., 2015a; Paixao et al., 2015b; Aprianto et al., 2018). Biofilm-dispersing Spn exhibit aggressive growth and virulence phenotypes through their increased production of bacteriocins, virulence factors (capsule, $c b p \mathrm{~A}$, pspA, ply, pcpA, nan $\mathrm{A}$, and nan $\mathrm{B}$ ), proteins of unknown function (SPV_2027 and SPV_2171), and factors related to carbohydrate metabolism, while reducing the expression of genes for competence and adhesion (Gualdi et al., 2012; Allan et al., 2014; Pettigrew et al., 2014; Paixao et al., 2015a; Paixao et al., 2015b; Aprianto et al., 2018). Particle matters (i.e., Asian sand dust, cigarette smoke and black carbon source) induce biofilm formation hence Spn colonization of the human ME epithelium in cell lines and in the ME of mouse OM model (Hussey et al., 2017; Yadav et al., 2020). Genes for biofilm formation $(\operatorname{lux} S)$, competence $(\operatorname{com} A, \operatorname{com} B, \operatorname{ciaR})$, toxin production (lytA and ply), detoxification, efflux pumps and osmo-regulator transporters were up-regulated during pneumococcal infection (Cockeran et al., 2014; Manna et al., 2018; Yadav et al., 2020).

\section{Mechanism in Gene Regulation: Pheromone Peptide Signaling}

Gene expression in Spn, in response to environmental stimuli, is regulated and synchronized at a population-level through quorum sensing or cell-cell communication systems that are orchestrated by short peptide pheromone-mediated signaling pathways. Three main types of pneumococcal cell-cell communication systems have been discovered so far. They are either mediated by (i) glycine-glycine (GG) peptides, (ii) peptides that signal via RRNPP superfamily regulators, or (iii) lanthionine-containing peptides, as depicted in Figure 2A. The most well-studied pneumococcal GG peptide signaling is the competence-stimulating peptide (CSP) that is autoinduced hence actively secreted when the surrounding $\mathrm{pH}$, hemoglobin, oxygen levels, antimicrobial stress, and, finally, the bacterial cell density is high (Gagne et al., 2013; Slager et al., 2014; Domenech et al.,
2018; Weyder et al., 2018; Paton and Trappetti, 2019; Akhter et al., 2021). A sufficient level of the GG peptide eventually signals the neighboring recipient pneumococci via a twocomponent system (TCS) and alter their gene expression. The CSP signaling is responsible for the induction of (i) competence and transformation in Spn contributing to genetic diversity or as nutrient source; (ii) bacteriocin peptides (CibAB, peptides of the bacteriocin immunity region (BIR), and bacteriocin-inducing peptide (BIP)) for microbial competition; and (iii) biofilmregulating peptide induced by competence (BriC) that induces the biofilm formation and nasopharyngeal colonization in animal models while altering the fatty acid biosynthesis in the pneumococcal membrane homeostasis for increased adaptation in the host (Valente et al., 2016; Wholey et al., 2016; Aggarwal et al., 2018; Wang et al., 2018; Aggarwal et al., 2021).

Due to a low availability of glucose in the airways, Spn in the nasopharynx and ME relies on the galactose and mannose derived from the airway mucosal glycan lining, as a main carbon source for energy metabolism and virulence (Paixao et al., 2015a; Paixao et al., 2015b). Several recent studies have unveiled the impact of carbohydrate utilization and metabolic processes in Spn virulence, persistence, and infection at nasopharynx, which involves a series of regulators (galK, galR, $h y l$, ugl, lacD, nanA, eng, rafK, estA, and auto-inducer AI-2) (Afzal et al., 2015; Mclean et al., 2020; Minhas et al., 2021). Sensing of the host carbohydrates for environmental adaptation is mainly carried out by pneumococcal short hydrophobic peptide (SHP) and phosphatase regulator (Phr) that interact with RRNPP superfamily of transcription regulator, regulator gene of glycosyltrasferase (Rgg) and transcription factor regulated by $\mathrm{Phr}$ peptide (Tpr), respectively. High abundance of galactose and mannose stimulates SHP144 and SHP939 to autoinduce, whereas induction of $\mathrm{PhrA}$ is more related to galactose dependence (Junges et al., 2017; Zhi et al., 2018; Motib et al., 2019). SHP144 and SHP939 imported into the recipient neighboring pneumococci positively regulate their cognate regulator, Rgg144 and Rgg939, respectively. This results in the upregulation of regulons involved in environmental adaptation (i.e., genes for replication and translation, nucleotide metabolism, cell division, ion transport, and capsule production) (Junges et al., 2017; Zhi et al., 2018; Cuevas et al., 2019). The SHP144/Rgg144 signaling also positively regulates the transcription of VP1, a novel virulent GG-peptide of Spn that was highly expressed in chinchilla ME effusions (Cuevas et al., 2017; Cuevas et al., 2019). VP1 activates biofilm development, colonization, hyaluronic acid-dependent attachment of Spn. On the other hand, PhrA imported into the recipient pneumococci inhibits the repressor role of its cognate regulator TprA, activating the transcription of genes for sugar metabolism, neuraminidase activity, and locus of lanthioninecontaining peptide for microbial competition during colonization (Hoover et al., 2015; Motib et al., 2017; Motib et al., 2019). Lantibiotic-containing peptides (pneumolancidins, Pld) are another class of pneumococcal bacteriocins that can be autoinduced by histidine kinase receptor signaling in response to the carbon source and cell density (Hoover et al., 
A

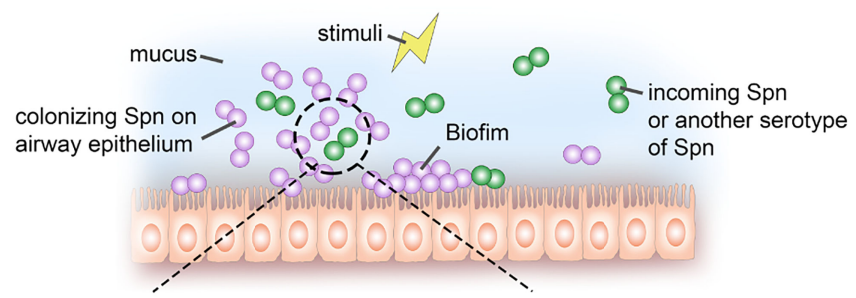

(a)

GG peptide signalling

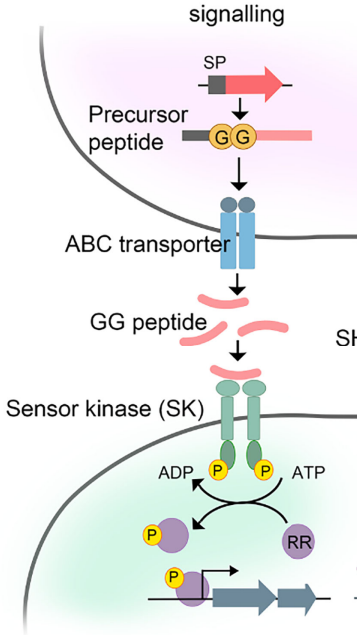

RRNPPdependent signalling

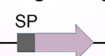

$\stackrel{\text { SP }}{\perp} \longrightarrow$
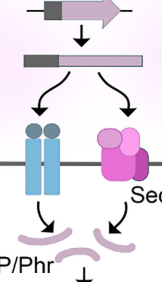
peptide signalling

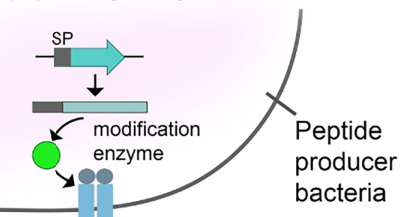
producer
bacteria

.

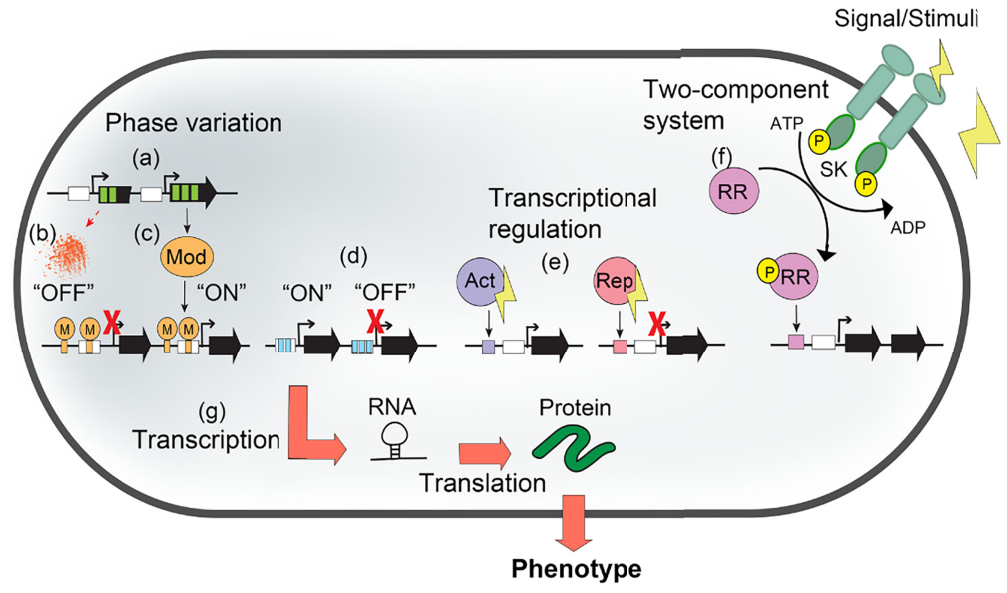

$\begin{array}{lll}\square-\text { Promoter } & \text { DNA-binding site for activator } \\ \text { D No transcription } & \text { DNA-binding site for repressor }\end{array}$

FIGURE 2 | Continued 
FIGURE 2 | Gene expression regulatory system in S. pneumoniae (Spn), and nontypeable H. influenzae (NTHi), and M. catarrhalis (Mcat). (A) Pneumococcal cell-cell communication systems. Spn colonizing the upper airway mucosa (i.e., at nasopharynx and middle ear) form biofilm and interact with either neighbouring pneumococcal or incoming clone of Spn via quorum sensing or cell-cell communication in response to surrounding stimuli (upper panel). Pheromone peptide signalling pathways and their regulatory effect in gene expression during Spn cell-cell communication are shown in the lower panel. Pheromone peptides such as double glycine peptides [GG peptide (i.e., CSP, BriC, VP1)] [as shown in pathway (a)], RRNPP-dependent peptides (i.e., SHR and Phr) [shown in (b)], and lanthionine peptides (i.e., pneumolancidin PldA1-A4) (shown in (c)) are secreted by pneumococci and autoinduced in response to stimuli. Depending on the signal sequence (SP) of the peptides, they are exported via ABC transporter or general secretory (SEC) pathway. The precursor peptide is proteolytically processed into active peptide either during transportation by peptidase domain of ABC transporter or membrane-associated proteases, or after secretion via unknown extracellular protease. At a sufficient level of the signalling peptide pheromone, they interact with their cognate cell receptors in recipient cells (peptide responder bacteria) such as sensor kinase (SK) of two-component system (TCS) [shown in pathway (a) and (c)]. In canonical TCS, the sensor protein is a histidine kinase (i.e., ComAB, BlpAB and PptAB) that detects exogenous signals, and subsequently, sends a phosphoryl group (yellow sphere) to the cognate response regulator protein (RR). The phosphorylation of the regulator protein results in transcriptional regulation. Alternatively, some peptides such as bacteriocin CibAB and lanthibiotics (pneumolandin) can activate recipient cell directly and induce bactericidal effect. As shown in pathway (b), for RRNPP-dependent peptides such as SHP (i.e., SHP144, SHP939 and RgtS) and Phr (i.e., PhrA), they are transported into responder cell via oligopeptide permease system (i.e., AmiACDEF). Once inside the recipient cell, SHPs interacts with their cognate binding partner, Rgg regulators (i.e., Rgg144, Rgg939, abd RgtR), resulting in DNA binding of Rgg and activation of transcription. On the other hand, Phr peptides interact with Tpr regulators that are initially bound to DNA and inhibit the expression of the target gene. Binding of Phr to Tpr results in the releasing of Tpr-mediated inhibition hence activates gene expression. Lastly, lanthionine-containing peptide such as pneumolandin (i.e., PldA1-A4) is processed and translationally modified by intracellular modification enzyme such as LanM before transportation via ABC transporter (i.e., LanT) and detected by SK of responder cells (i.e., LanA) [shown in pathway (c)]. This results in either phosphorylation of the response regulator for downstream activation of gene expression, or directly causing bactericidal effect. ABC transporter, ATP-binding cassette transporter; Phr, phosphatase regulator; Rgg, regulator gene of glycosyltransferase; RRNPP, Rap, Rgg, NprC, PlcR and PrgX; SHP, short hydrophobic peptide. (B) Schematic representation of gene regulatory mechanism in NTHi and Mcat. Phase variation [shown in (a)] is caused by random mutations in a variable number of simple sequence repeats (SSR) within the open reading frame of DNA-methyl transferases (i.e., ModA and ModM] that alters the gene expression of Mod. Thus, any changes in the variable number of the SSRs as a result of DNA mutation could cause frame shifts in the ORF of modA and potentially lead to premature translation termination and generation of non-functional truncated ModA which is in the "OFF" mode (shown in (b)]. On the other hand, the functional Mod (switched "ON") [shown in (c)] methylates genomic DNA at specific sites that governs transcription in both ways, either by inhibition or activation. The inhibition or activation of transcription depends on the methylated DNA sequence area that potentially bears the recognition sites for regulatory molecules and enzymes. Moreover, the phase-variable number of SSRs [as shown in (d)] can also be found in the transcriptional promoter which, as a result, switches between the "ON" or "OFF" expression status of the target gene (i.e., hia). In addition, transcriptional regulators [shown in (e)] can be activators (Act) (i.e., OxyR, Fur) and/or repressors (Rep) (i.e., Fur) of transcription. The regulators are triggered by various stimuli. (f) Another type of transcriptional regulation is a TCS (i.e., FirRS, mesSR, narX/narL) which consists of sensor kinase (SK) and response regulator proteins (RR). The activated regulatory proteins can cause transcription activation and/or repression as described in (e). Finally [indicated in (g)], the genes are transcribed into RNA, followed by translation as protein. Alterations in gene expression are manifested in a bacterial phenotype. Symbols used in panel $\mathbf{( A , B )}$ are defined at the bottom of this figure.

2015; Maricic et al., 2016). This ultimately benefit pneumococci to outcompete co-colonizing bacteria for space and nutrients during colonization.

LuxS/AI-2-dependent quorum sensing system is also another crucial gene regulatory system in Spn especially for biofilm formation, genetic competence, and fratricide (Trappetti et al., 2011; Yadav et al., 2018). The transcriptomic and mutagenesis studies revealed that LuxS is the central regulator of genes important for Spn virulence and persistence in carriage phase and middle ear infections.

\section{Non-Typeable Haemophilus influenzae}

Non-typeable Haemophilus influenzae (NTHi) is a Gramnegative coccobacillus without capsule that is genetically nonclonal (Erwin et al., 2005; Whittaker et al., 2017). NTHi is part of the nasopharyngeal commensal microbiota. The bacterial species can also, however, cause airway infections such as OM. The currently used vaccine specific to $H$. influenzae type b (Hib) is not effective against NTHi. The pathogen is heme and nicotinamide adenine dinucleotide (NAD) auxotroph, hence requires exogenous supplementation of these elements for growth (Jalalvand and Riesbeck, 2018; Su et al., 2018).

\section{Gene Expression During Colonization}

Similarly to Spn, NTHi colonizing the human airway needs to overcome the harsh environmental conditions in the host in order to establish a fulminant infection. Proteomic analysis on
NTHi infecting the chinchilla ME revealed the altered expression of 28 bacterial proteins involved in carbohydrate and amino acid metabolism, redox homeostasis, and cell wall-associated metabolic proteins (Harrison et al., 2016). This implies the utilization of glucose by NTHi for aerobic respiration during animal AOM.

Inflammation triggered in the ME upon infection leads to activation of nutritional immunity (Szelestey et al., 2013). This results in the sequestration and restriction of free metal ions available for NTHi iron/heme-dependent metabolic enzymes, regulatory proteins, and aerobic respiration that are essential for bacterial ME colonization (Szelestey et al., 2013; Harrison et al., 2016). Since NTHi is heme-iron auxotroph, the pathogen develops a plethora of iron/heme acquisition mechanisms in response to the host nutritional immunity. This includes the upregulation and expression of a series of core iron- and hemeresponsive genes which some are regulated by the NTHi ferric uptake regulator (Fur) (Figure 2B) and RNA chaperon Hfq (Harrison et al., 2013; Hempel et al., 2013; Whitby et al., 2013). This in turn promotes the colonization persistence and virulence factor expression in NTHi when infecting the chinchilla ME. Besides the iron uptake genes, Fur also regulates the transcription of small RNA HrrF that is important for molybdate uptake, deoxyribonucleotide synthesis and amino acid biosynthesis (Santana et al., 2014). The high abundance of iron/heme also activates the promoter activity of pilA, hence the expression of subunit PilA for the type IV pilus that is essential 
for NTHi adherence and biofilm formation (Mokrzan et al., 2019). Moreover, the co-culture with Spn enhances pilA expression in NTHi and biofilm formation as well (Cope et al., 2011). Biofilm formation is crucial for NTHi colonization in OM, and the biofilm is also regulated by a TCS transduction system named QseBC/FirRS (ferrous iron responsive regulator/sensor) (Figure 2B), which is responsive to low temperature and availability of nutrients (ferrous iron and zinc) (Steele et al., 2012; Unal et al., 2012; Van Hoecke et al., 2016). In addition, high $\mathrm{pH}$ in the $\mathrm{ME}$ during $\mathrm{OM}$ promotes biofilm formation and expression of virulence factors for iron acquisition (Ishak et al., 2014).

Beside the nutritional immunity, NTHi also needs to combat oxidative stress such as reactive oxygen species (ROS) and reactive nitrogen species (RNS) generated by the airway immune defense. Hence, NTHi upregulated the expression of OxyR regulon (antioxidant enzymes (peroxiredoxin (PgdX) and catalase (HktE)) as shown in the chinchilla ME (Whitby et al., 2012; Parrish et al., 2019).

\section{Mechanism in Gene Regulation: Phase Variation}

Phase variation is one of the most studied gene regulation mechanisms in NTHi. It is widely used by NTHi to regulate the expression of virulence factors that are crucial for bacterial colonization at specific niches. Phase variation is a random molecular event that enable a specific gene expression to be reversibly switched "ON" and "OFF". This mechanism is based upon the DNA mutation that results in the formation of variable number of simple sequence repeats (SSR) in the genome (Srikhanta et al., 2005) (Figure 2B).

In NTHi, N6-adenine DNA-methyltransferase (ModA) is one of the proteins that have a phase variable expression. The enzyme which is part of the type III restriction-modification (R-M) system methylates bacterial chromosomal DNA at a specific site on the genome, mediating epigenetic regulation (Gawthorne et al., 2012). ModA is encoded by a modA allele. Up to 21 allelic variants of $\operatorname{modA}(\bmod A 1-21)$ exist in NTHi clinical isolates taken from COPD patients and children with ME infection or OM (Atack et al., 2015a; Atack et al., 2019). However, among the identified modA allelic variants, $65 \%$ of the isolates carry one of just 5 phase-variable modAs, namely allelic variant of $\operatorname{modA2}, 4$, 5, 9, or 10 (Fox et al., 2007; Atack et al., 2015a). These alleles contain different numbers of SSRs and hence can alter the expression of ModA. The phase variation of these alleles impacts (i) the gene expression of (a) known NTHi virulence major outer membrane proteins (P2, P5, P6, and HMW) $(\bmod A 2,4,5,9$, and 10-dependence) and (b) NTHi proteins involved in antibiotic resistance $(\bmod A 2,5$ and 10$)$, and (ii) evasion of opsonophagocytic killing $(\operatorname{modA} 4)$.

In response to the alkaline $\mathrm{pH}$ environment occurred in the ME during OM, ModA2 of NTHi mediates the formation of a bacterial biofilm that has greater biomass and stable structure which are critical for NTHi pathogenesis in vivo (Atack et al., 2015a; Brockman et al., 2018). NTHi with phase variable modA2 expression (reversibly switch between "ON" and "OFF") has survival and adaptational advantages for colonization at chinchilla ME over an isogenic strain that is unable to phase vary their ModA2 expression. This is explained by the fact that NTHi carrying modA2 that is permanently "OFF" or "ON" is more susceptible to macrophage and neutrophil killing, respectively; whereas strains carrying phase variable modA2 (switchable from "OFF" to "ON") may alter their virulence factor expression for increased antigenic variation hence evasion of the host defense (Brockman et al., 2016; RobledoAvila et al., 2020). Collectively, the methylation-dependent gene regulation mediated by modA influences the virulence of NTHi especially during infection at ME. In addition, to avoid the host immune system during invasive infection, NTHi also alters the expression of Hia, an immunogenic adhesin, by changing the length of polythymidine tract on the hia promoter (Atack et al., 2015b). SSR-dependent phase variation is also used by NTHi for persistence and adaptation in the pathogenesis of other airway infections such as COPD (Poole et al., 2013; Pettigrew et al., 2018; Fernandez-Caldet et al., 2021).

\section{Moraxella catarrhalis}

Moraxella catarrhalis is a Gram-negative respiratory opportunistic pathogen. It is categorized into two distinct lineages based on $16 \mathrm{~S}$ rRNA sequence: (i) ribotype (RB) 1 which comprise of $\sim 80-90 \%$ of isolates associated with adherence and serum resistance; (ii) RB2 and RB3 strains that are detected in $\sim 10-20 \%$ isolates (Bootsma et al., 2000; Verhaegh et al., 2011).

\section{Gene Expression During Colonization}

Regulation in gene expression of virulence factors is crucial for Mcat colonization and long-term survival within the host in the human airways. There are a range of extrinsic stimuli that this pathogen must contend with, such as low temperature and iron restricted conditions within the human airway. The environmental factors affect the expression of Mcat major outer membrane proteins that are the key to efficient colonization (Jetter et al., 2010; Spaniol et al., 2011). Notably, genes of resistance-nodulation-division (RND) multidrug efflux systems (acr $\mathrm{AB}$ and $o p r \mathrm{M})$ are upregulated at nasopharyngeal temperature $\left(26^{\circ} \mathrm{C}\right)$ (Spaniol et al., 2013). Deletion of $a c r A B$ and opr $\mathrm{M}$ caused Mcat to lose up to $50 \%$ invasion capacity on human pharyngeal epithelial cells (Spaniol et al., 2015).

PilA that mediates Mcat in vivo colonization in the chinchilla model was the earliest adhesin to be investigated (Luke et al., 2007). Hoopman et al., further elucidated the Mcat colonization via DNA microarray analysis which showed the upregulated genes involved in the oxidative stress response, denitrification pathway, in addition to an uncharacterized gene (ORF1550) that is crucial for Mcat persistence (Hoopman et al., 2012). Other well-known adhesin-encoding virulence genes, such as uspA1, uspA2, uspA2H, mid/hag and mcaP, mha/mch and $m c m$ also play important roles in protecting Mcat from the serum bactericidal effect by interfering with the host complement pathway factors (Singh et al., 2016; Riesbeck, 2020). A mutation study revealed the impact of an outer membrane lipoprotein (ORF113) on the survival of Mcat in chinchilla (Wang et al., 2014). 
Several Mcat virulence factors are growth phase dependent. The strain Mcat RH4 displayed increased expression of mid/hag during the lag and stationary phases but fell to lower levels during exponential phase (Riesbeck and Nordstrom, 2006; De Vries et al., 2010). MID/Hag protein is probably essential for initial colonization but not for proliferation. In a separate study, the downregulated expression of mid/hag and uspA2 occurred during infection in chinchilla (Hoopman et al., 2012). MID/Hag expression was also reduced during persistent colonization of Mcat in COPD patients (Murphy et al., 2019). It appears that reducing the profusion of surface proteins could aid Mcat to avoid host recognition.

One of the main modes of Mcat colonizing the ME during infection is via biofilms. The synergistic relationship Mcat has with its co-colonizing pathogens increases OM incidence (Blakeway et al., 2018). Mcat secretes beta-lactamasecontaining outer membrane vesicles (OMVs) in biofilms which protects co-colonizing pathogens from beta-lactam antibiotics (Armbruster et al., 2010; Schaar et al., 2013). There are interesting facets of biofilm formation which include the quorum-sensing systems found in Spn, NTHi and Mcat. Armbruster et al. established the compensation effect of the $\mathrm{NTHi}$ luxS gene (interspecies quorum signaling factor) in aiding Mcat for persistence in chinchilla since the latter species does not possess a luxS homolog (Armbruster et al., 2010; Mokrzan et al., 2018).

\section{Mechanism in Gene Regulation: Phase Variation and Two-Component System}

Once Mcat has colonized the host, bacteria regulate the expression of its cell surface components by phase variation. The phase variation mechanism that has been widely studied in Mcat is performed by several DNA methyltransferases including ModM. The DNA methyltransferases are part of R-M system that master mind the gene regulation in Mcat during infection (Blakeway et al., 2018; Phillips et al., 2019) (Figure 2B).

Similar to NTHi, Mcat also carries multiple allelic variants of $\bmod \mathrm{M}(\bmod \mathrm{M} 1-6)$. The most common allele of $\bmod \mathrm{M}$ present in Mcat isolates is $\bmod \mathrm{M} 2$ which regulates genes associated with colonization and host immune evasion (Blakeway et al., 2019). Although the $\operatorname{modM} 3$ allele is commonly found within the minor lineage RB2 and RB3 of Mcat strains, the ModM3 methyltransferase is prevalent in Mcat clinical isolates taken from children with OM (Blakeway et al., 2014). Extensive methodologies were used including single molecular real-time (SMRT) methylome analysis to ascertain the methylation activity of ModM3. Also, RNASeq analysis revealed that ModM3 alters the expression of genes involved in biofilm formation, anaerobic tolerance, nitrosative and oxidative stress responses. Specifically, in response to host immune defense-derived nitrosative and oxidative stress, Mcat with "ON" variant of modM3 upregulated the expression of genes narX/narL, and a predicted AhpC/TSA family peroxiredoxin RS03200, respectively (Blakeway et al., 2019). Additionally, in a recent study aiming to reveal the correlation between serum resistance and phase variation, Mcat strains that survived in human serum have higher mRNA levels of uspA2 whereas mid/hag and uspA1 showed reduced expression over a longer time of exposure to the human serum (Tan et al., 2020). Under this selection pressure, variable SSR tract lengths that regulate transcription hence phase variation in uspA1, uspA2 and mid/hag is likely to occur. Antigenic variation of outer membrane proteins is another strategy of Mcat for host immune evasion (Murphy et al., 2019; Tan et al., 2020).

Another gene regulatory mechanism of Mcat is the TCS signaling systems (Figure 2B). mesR is a gene of this signalling system, and potentially regulates expression of two lysozyme inhibitor genes as a defence against the host immune response (Joslin et al., 2015). Another example of TCS is the narX/narL involved in denitrification pathway. This system counteracts reactive nitrogen species produced exogenously and could possibly ensure Mcat survival under anaerobic conditions (Blakeway et al., 2019).

\section{CONCLUSION}

The ability to overcome environmental challenges during colonization inside the host is crucial for bacterial survival and persistence, hence establishment of infection. Spn senses the environmental stimuli and regulates its gene expression through short peptide pheromone-mediated signalling pathways and LuxS/ AI-2-dependent quorum sensing system. This enables Spn to regulate the switch between biofilm and planktonic phenotype for colonization or invasion, respectively; genetic competence, and fratricide activities, and expression of regulons involved in environmental adaptation and evasion of host immune system. On the other hand, in addition to transcriptional regulators, gene regulation in NTHi and Mcat is mainly mediated via phase variation mechanisms that reversibly switches the expression "ON" or "OFF" of the target genes. The system is based on the presence of the variable number of SSRs, as a result of slippedstrand mispairing that occurred either on the promoter or within the ORF, or both, of the target gene. This enables NTHi and Mcat to regulate their biofilm formation, adherence, serum resistance and other virulence phenotype according to growth phase or in response to selective pressure caused by host immune response. The findings discussed in this review could help to improve our understanding regarding the gene expression governing system in these pathogens and can be a potential target of future antimicrobial intervention strategy against Spn, NTHi and Mcat infection.

\section{AUTHOR CONTRIBUTIONS}

MJ coordinated the manuscript. MJ, MS, and Y-CS drafted the manuscript. Literature studies of virulence and gene regulation in NTHi, M. catarrhalis and S. pneumoniae were carried out by MJ, MS, and Y-CS, respectively. Figures were mainly prepared by MJ and MS assisted by Y-CS. All authors edited, critically revised, and approved the final manuscript. 


\section{FUNDING}

We thank the following funding agencies for their financial support during the preparation of the manuscript. They are, the Swedish Medical Research Council (KR: grant number 2019-

\section{REFERENCES}

Afzal, M., Shafeeq, S., Manzoor, I., and Kuipers, O. P. (2015). GalR Acts as a Transcriptional Activator of galKT in the Presence of Galactose in Streptococcus Pneumoniae. J. Mol. Microbiol. Biotechnol. 25 (6), 363-371. doi: 10.1159/000439429

Aggarwal, S. D., Eutsey, R., West-Roberts, J., Domenech, A., Xu, W., Abdullah, I. T., et al. (2018). Function of BriC Peptide in the Pneumococcal Competence and Virulence Portfolio. PloS Pathog. 14 (10), e1007328. doi: 10.1371/ journal.ppat.1007328

Aggarwal, S. D., Gullett, J. M., Fedder, T., Safi, J. P. F., Rock, C. O., and Hiller, N. L. (2021). Competence-Associated Peptide BriC Alters Fatty Acid Biosynthesis in Streptococcus pneumoniae. mSphere 6 (3), e0014521. doi: 10.1128/ $\mathrm{mSphere.00145-21}$

Akhter, F., Womack, E., Vidal, J. E., Le Breton, Y., Mciver, K. S., Pawar, S., et al. (2021). Hemoglobin Induces Early and Robust Biofilm Development in Streptococcus Pneumoniae by a Pathway That Involves comC But Not the Cognate comDE Two-Component System. Infect. Immun. 89 (4), e00779-20. doi: 10.1128/IAI.00779-20

Allan, R. N., Skipp, P., Jefferies, J., Clarke, S. C., Faust, S. N., Hall-Stoodley, L., et al. (2014). Pronounced Metabolic Changes in Adaptation to Biofilm Growth by Streptococcus Pneumoniae. PloS One 9 (9), e107015. doi: 10.1371/ journal.pone.0107015

Aprianto, R., Slager, J., Holsappel, S., and Veening, J. W. (2018). High-Resolution Analysis of the Pneumococcal Transcriptome Under a Wide Range of Infection-Relevant Conditions. Nucleic Acids Res. 46 (19), 9990-10006. doi: 10.1093/nar/gky750

Armbruster, C. E., Hong, W., Pang, B., Weimer, K. E., Juneau, R. A., Turner, J., et al. (2010). Indirect Pathogenicity of Haemophilus Influenzae and Moraxella Catarrhalis in Polymicrobial Otitis Media Occurs via Interspecies Quorum Signaling. mBio 1 (3), e00102-00110. doi: 10.1128/mBio.00102-10

Atack, J. M., Murphy, T. F., Pettigrew, M. M., Seib, K. L., and Jennings, M. P. (2019). Non-Typeable Haemophilus Influenzae Isolates From Patients With Chronic Obstructive Pulmonary Disease Contain New Phase-Variable modA Methyltransferase Alleles Controlling Phasevarions. Sci. Rep. 9 (1), 15963. doi: 10.1038/s41598-019-52429-6

Atack, J. M., Srikhanta, Y. N., Fox, K. L., Jurcisek, J. A., Brockman, K. L., Clark, T. A., et al. (2015a). A Biphasic Epigenetic Switch Controls Immunoevasion, Virulence and Niche Adaptation in Non-Typeable Haemophilus Influenzae. Nat. Commun. 6, 7828. doi: 10.1038/ncomms 8828

Atack, J. M., Winter, L. E., Jurcisek, J. A., Bakaletz, L. O., Barenkamp, S. J., and Jennings, M. P. (2015b). Selection and Counterselection of Hia Expression Reveals a Key Role for Phase-Variable Expression of Hia in Infection Caused by Nontypeable Haemophilus Influenzae. J. Infect. Dis. 212 (4), 645-653. doi: 10.1093/infdis/jiv103

Biesbroek, G., Tsivtsivadze, E., Sanders, E. A., Montijn, R., Veenhoven, R. H., Keijser, B. J., et al. (2014). Early Respiratory Microbiota Composition Determines Bacterial Succession Patterns and Respiratory Health in Children. Am. J. Respir. Crit. Care Med. 190 (11), 1283-1292. doi: 10.1164/ rccm.201407-1240OC

Blakeway, L. V., Power, P. M., Jen, F. E., Worboys, S. R., Boitano, M., Clark, T. A., et al. (2014). ModM DNA Methyltransferase Methylome Analysis Reveals a Potential Role for Moraxella Catarrhalis Phasevarions in Otitis Media. FASEB J. 28 (12), 5197-5207. doi: 10.1096/fj.14-256578

Blakeway, L. V., Tan, A., Jurcisek, J. A., Bakaletz, L. O., Atack, J. M., Peak, I. R., et al. (2019). The Moraxella Catarrhalis Phase-Variable DNA Methyltransferase ModM3 is an Epigenetic Regulator That Affects Bacterial Survival in an In Vivo Model of Otitis Media. BMC Microbiol. 19 (1), 276. doi: 10.1186/s12866-019-1660-y
01053, www.vr.se), the Anna and Edwin Berger Foundation (KR), the Physiographical Society (Forssman's Foundation; MJ, MS), Skåne County Council's research and development foundation (KR), and Heart Lung Foundation (KR: grant number 20180401, www.hjart-lungfonden.se).

Blakeway, L. V., Tan, A., Lappan, R., Ariff, A., Pickering, J. L., Peacock, C. S., et al. (2018). Moraxella Catarrhalis Restriction-Modification Systems Are Associated With Phylogenetic Lineage and Disease. Genome Biol. Evol. 10 (11), 2932-2946. doi: 10.1093/gbe/evy226

Bootsma, H. J., van der Heide, H. G., Van De Pas, S., Schouls, L. M., and Mooi, F. R. (2000). Analysis of Moraxella Catarrhalis by DNA Typing: Evidence for a Distinct Subpopulation Associated With Virulence Traits. J. Infect. Dis. 181 (4), 1376-1387. doi: $10.1086 / 315374$

Brockman, K. L., Azzari, P. N., Branstool, M. T., Atack, J. M., Schulz, B. L., Jen, F. E., et al. (2018). Epigenetic Regulation Alters Biofilm Architecture and Composition in Multiple Clinical Isolates of Nontypeable Haemophilus Influenzae. mBio 9 (5), e01682-18. doi: 10.1128/mBio.01682-18

Brockman, K. L., Jurcisek, J. A., Atack, J. M., Srikhanta, Y. N., Jennings, M. P., and Bakaletz, L. O. (2016). ModA2 Phasevarion Switching in Nontypeable Haemophilus influenzae Increases the Severity of Experimental Otitis Media. J. Infect. Dis. 214 (5), 817-824. doi: 10.1093/infdis/jiw243

Brooks, L. R. K., and Mias, G. I. (2018). Streptococcus Pneumoniae's Virulence and Host Immunity: Aging, Diagnostics, and Prevention. Front. Immunol. 9. doi: 10.3389/fimmu.2018.01366

Chao, Y., Marks, L. R., Pettigrew, M. M., and Hakansson, A. P. (2014). Streptococcus Pneumoniae Biofilm Formation and Dispersion During Colonization and Disease. Front. Cell Infect. Microbiol. 4. doi: 10.3389/fcimb.2014.00194

Cockeran, R., Herbert, J. A., Mitchell, T. J., Dix-Peek, T., Dickens, C., Anderson, R., et al. (2014). Exposure of a 23F Serotype Strain of Streptococcus Pneumoniae to Cigarette Smoke Condensate is Associated With Selective Upregulation of Genes Encoding the Two-Component Regulatory System 11 (Tcs11). BioMed. Res. Int. 2014, 976347. doi: 10.1155/2014/976347

Coleman, A., Wood, A., Bialasiewicz, S., Ware, R. S., Marsh, R. L., and Cervin, A (2018). The Unsolved Problem of Otitis Media in Indigenous Populations: A Systematic Review of Upper Respiratory and Middle Ear Microbiology in Indigenous Children With Otitis Media. Microbiome 6 (1), 199. doi: 10.1186/ s40168-018-0577-2

Cope, E. K., Goldstein-Daruech, N., Kofonow, J. M., Christensen, L., Mcdermott, B., Monroy, F., et al. (2011). Regulation of Virulence Gene Expression Resulting From Streptococcus Pneumoniae and Nontypeable Haemophilus Influenzae Interactions in Chronic Disease. PloS One 6 (12), e28523. doi: 10.1371/journal.pone.0028523

Cuevas, R. A., Ebrahimi, E., Gazioglu, O., Yesilkaya, H., and Hiller, N. L. (2019). Pneumococcal Attachment to Epithelial Cells is Enhanced by the Secreted Peptide VP1 via its Control of Hyaluronic Acid Processing. bioRxiv [Preprint], 788430. doi: $10.1101 / 788430$

Cuevas, R. A., Eutsey, R., Kadam, A., West-Roberts, J. A., Woolford, C. A., Mitchell, A. P., et al. (2017). A Novel Streptococcal Cell-Cell Communication Peptide Promotes Pneumococcal Virulence and Biofilm Formation. Mol. Microbiol. 105 (4), 554-571. doi: 10.1111/mmi.13721

De Vries, S. P., Van Hijum, S. A., Schueler, W., Riesbeck, K., Hays, J. P., Hermans, P. W., et al. (2010). Genome Analysis of Moraxella Catarrhalis Strain BBH18, [Corrected] a Human Respiratory Tract Pathogen. J. Bacteriol. 192 (14), 3574 3583. doi: 10.1128/JB.00121-10

Domenech, A., Slager, J., and Veening, J. W. (2018). Antibiotic-Induced Cell Chaining Triggers Pneumococcal Competence by Reshaping Quorum Sensing to Autocrine-Like Signaling. Cell Rep. 25 (9), 2390-2400.e2393. doi: 10.1016/ j.celrep.2018.11.007

Erwin, A. L., Nelson, K. L., Mhlanga-Mutangadura, T., Bonthuis, P. J., Geelhood, J. L., Morlin, G., et al. (2005). Characterization of Genetic and Phenotypic Diversity of Invasive Nontypeable Haemophilus Influenzae. Infect. Immun. 73 (9), 5853-5863. doi: 10.1128/IAI.73.9.5853-5863.2005

Fernandez-Caldet, A., Euba, B., Gil-Campillo, C., Catalan-Moreno, A., Moleres, J., Marti, S., et al. (2021). Phase Variation in HMW1A Controls a Phenotypic 
Switch in Haemophilus Influenzae Associated With Pathoadaptation During Persistent Infection. mBio 12 (3), e0078921. doi: 10.1128/mBio. 00789-21

Fox, K. L., Dowideit, S. J., Erwin, A. L., Srikhanta, Y. N., Smith, A. L., and Jennings, M. P. (2007). Haemophilus Influenzae Phasevarions Have Evolved From Type III DNA Restriction Systems Into Epigenetic Regulators of Gene Expression. Nucleic Acids Res. 35 (15), 5242-5252. doi: 10.1093/nar/gkm571

Gagne, A. L., Stevens, K. E., Cassone, M., Pujari, A., Abiola, O. E., Chang, D. J., et al. (2013). Competence in Streptococcus Pneumoniae is a Response to an Increasing Mutational Burden. PloS One 8 (8), e72613. doi: 10.1371/ journal.pone. 0072613

Gawthorne, J. A., Beatson, S. A., Srikhanta, Y. N., Fox, K. L., and Jennings, M. P. (2012). Origin of the Diversity in DNA Recognition Domains in Phasevarion Associated modA Genes of Pathogenic Neisseria and Haemophilus influenzae. PloS One 7 (3), e32337. doi: 10.1371/journal.pone.0032337

Gualdi, L., Hayre, J. K., Gerlini, A., Bidossi, A., Colomba, L., Trappetti, C., et al. (2012). Regulation of Neuraminidase Expression in Streptococcus Pneumoniae. BMC Microbiol. 12, 200. doi: 10.1186/1471-2180-12-200

Harrison, A., Dubois, L. G., St John-Williams, L., Moseley, M. A., Hardison, R. L., Heimlich, D. R., et al. (2016). Comprehensive Proteomic and Metabolomic Signatures of Nontypeable Haemophilus Influenzae-Induced Acute Otitis Media Reveal Bacterial Aerobic Respiration in an Immunosuppressed Environment. Mol. Cell Proteomics. 15 (3), 1117-1138. doi: 10.1074/ mcp.M115.052498

Harrison, A., Santana, E. A., Szelestey, B. R., Newsom, D. E., White, P., and Mason, K. M. (2013). Ferric Uptake Regulator and its Role in the Pathogenesis of Nontypeable Haemophilus influenzae. Infect. Immun. 81 (4), 1221-1233. doi: 10.1128/IAI.01227-12

Hempel, R. J., Morton, D. J., Seale, T. W., Whitby, P. W., and Stull, T. L. (2013). The Role of the RNA Chaperone Hfq in Haemophilus Influenzae Pathogenesis. BMC Microbiol. 13, 134. doi: 10.1186/1471-2180-13-134

Hendrixson, D. R., and St Geme, J. W.3rd (1998). The Haemophilus Influenzae Hap Serine Protease Promotes Adherence and Microcolony Formation, Potentiated by a Soluble Host Protein. Mol. Cell. 2 (6), 841-850. doi: 10.1016/s1097-2765(00)80298-1

Hoopman, T. C., Liu, W., Joslin, S. N., Pybus, C., Sedillo, J. L., Labandeira-Rey, M., et al. (2012). Use of the Chinchilla Model for Nasopharyngeal Colonization to Study Gene Expression by Moraxella catarrhalis. Infect. Immun. 80 (3), 982995. doi: 10.1128/IAI.05918-11

Hoover, S. E., Perez, A. J., Tsui, H. C., Sinha, D., Smiley, D. L., Dimarchi, R. D., et al. (2015). A New Quorum-Sensing System (TprA/PhrA) for Streptococcus Pneumoniae D39 That Regulates a Lantibiotic Biosynthesis Gene Cluster. Mol. Microbiol. 97 (2), 229-243. doi: 10.1111/mmi.13029

Hussey, S. J. K., Purves, J., Allcock, N., Fernandes, V. E., Monks, P. S., Ketley, J. M., et al. (2017). Air Pollution Alters Staphylococcus Aureus and Streptococcus Pneumoniae Biofilms, Antibiotic Tolerance and Colonisation. Environ. Microbiol. 19 (5), 1868-1880. doi: 10.1111/1462-2920.13686

Ishak, N., Tikhomirova, A., Bent, S. J., Ehrlich, G. D., Hu, F. Z., and Kidd, S. P. (2014). There is a Specific Response to $\mathrm{pH}$ by Isolates of Haemophilus Influenzae and This has a Direct Influence on Biofilm Formation. BMC Microbiol. 14, 47. doi: 10.1186/1471-2180-14-47

Jalalvand, F., and Riesbeck, K. (2018). Update on Non-Typeable Haemophilus Influenzae-Mediated Disease and Vaccine Development. Expert Rev. Vaccines 17 (6), 503-512. doi: 10.1080/14760584.2018.1484286

Jetter, M., Spaniol, V., Troller, R., and Aebi, C. (2010). Down-Regulation of Porin M35 in Moraxella Catarrhalis by Aminopenicillins and Environmental Factors and its Potential Contribution to the Mechanism of Resistance to Aminopenicillins. J. Antimicrob. Chemother. 65 (10), 2089-2096. doi: $10.1093 / \mathrm{jac} / \mathrm{dkq} 312$

Jiang, Z., Nagata, N., Molina, E., Bakaletz, L. O., Hawkins, H., and Patel, J. A. (1999). Fimbria-Mediated Enhanced Attachment of Nontypeable Haemophilus Influenzae to Respiratory Syncytial Virus-Infected Respiratory Epithelial Cells. Infect. Immun. 67 (1), 187-192. doi: 10.1128/IAI.67.1.187-192.1999

Joslin, S. N., Pybus, C., Labandeira-Rey, M., Evans, A. S., Attia, A. S., Brautigam, C. A., et al. (2015). A Moraxella Catarrhalis Two-Component Signal Transduction System Necessary for Growth in Liquid Media Affects Production of Two Lysozyme Inhibitors. Infect. Immun. 83 (1), 146-160. doi: 10.1128/IAI.02486-14
Jossart, G. H., Canafax, D. M., Erdmann, G. R., Lovdahl, M. J., Russlie, H. Q., Juhn, S. K., et al. (1994). Effect of Streptococcus Pneumoniae and Influenza A Virus on Middle Ear Antimicrobial Pharmacokinetics in Experimental Otitis Media. Pharm. Res. 11 (6), 860-864. doi: 10.1023/a:1018933925707

Junges, R., Salvadori, G., Shekhar, S., Amdal, H. A., Periselneris, J. N., Chen, T., et al. (2017). A Quorum-Sensing System That Regulates Streptococcus Pneumoniae Biofilm Formation and Surface Polysaccharide Production. mSphere 2 (5), e00324-17. doi: 10.1128/mSphere.00324-17

Kloosterman, T. G., and Kuipers, O. P. (2011). Regulation of Arginine Acquisition and Virulence Gene Expression in the Human Pathogen Streptococcus Pneumoniae by Transcription Regulators ArgR1 and AhrC. J. Biol. Chem. 286 (52), 44594-44605. doi: 10.1074/jbc.M111.295832

Lee, J., Kim, K. H., Jo, D. S., Ma, S. H., Kim, J. H., Kim, C. S., et al. (2020). A Longitudinal Hospital-Based Epidemiology Study to Assess Acute Otitis Media Incidence and Nasopharyngeal Carriage in Korean Children Up to 24 Months. Hum. Vaccin. Immunother. 16 (12), 3090-3097. doi: 10.1080/ 21645515.2020.1748978

Littorin, N., Ahl, J., Uddén, F., Resman, F., and Riesbeck, K. (2016). Reduction of Streptococcus Pneumoniae in Upper Respiratory Tract Cultures and a Decreased Incidence of Related Acute Otitis Media Following Introduction of Childhood Pneumococcal Conjugate Vaccines in a Swedish County. BMC Infect. Dis. 16 (1), 407. doi: 10.1186/s12879-016-1750-5

Littorin, N., Rünow, E., Ahl, J., Resman, F., and Riesbeck, K. (2021). Decreased Prevalence of Moraxella Catarrhalis in Addition to Streptococcus Pneumoniae in Children With Upper Respiratory Tract Infection After Introduction of Conjugated Pneumococcal Vaccine: A Retrospective Cohort Study. Clin. Microbiol. Infect. 27 (4), 630.e631-630.e636. doi: 10.1016/j.cmi.2020.04.033

Luke, N. R., Jurcisek, J. A., Bakaletz, L. O., and Campagnari, A. A. (2007). Contribution of Moraxella Catarrhalis Type IV Pili to Nasopharyngeal Colonization and Biofilm Formation. Infect. Immun. 75 (12), 5559-5564. doi: 10.1128/IAI.00946-07

Man, W. H., De Steenhuijsen Piters, W. A., and Bogaert, D. (2017). The Microbiota of the Respiratory Tract: Gatekeeper to Respiratory Health. Nat. Rev. Microbiol. 15 (5), 259-270. doi: 10.1038/nrmicro.2017.14

Manna, S., Waring, A., Papanicolaou, A., Hall, N. E., Bozinovski, S., Dunne, E. M., et al. (2018). The Transcriptomic Response of Streptococcus Pneumoniae Following Exposure to Cigarette Smoke Extract. Sci. Rep. 8 (1), 15716. doi: 10.1038/s41598-018-34103-5

Manzoor, I., Shafeeq, S., Kloosterman, T. G., and Kuipers, O. P. (2015). Co(2 + )-Dependent Gene Expression in Streptococcus Pneumoniae: Opposite Effect of $\mathrm{Mn}(2+)$ and $\mathrm{Co}(2+)$ on the Expression of the Virulence Genes psaBCA, Pcpa, and prtA. Front. Microbiol. 6. doi: 10.3389/fmicb.2015.00748

Maricic, N., Anderson, E. S., Opipari, A. E., Yu, E. A., and Dawid, S. (2016). Characterization of a Multipeptide Lantibiotic Locus in Streptococcus Pneumoniae. mBio 7 (1), e01656-e01615. doi: 10.1128/mBio.01656-15

Marks, L. R., Davidson, B. A., Knight, P. R., and Hakansson, A. P. (2013). Interkingdom Signaling Induces Streptococcus Pneumoniae Biofilm Dispersion and Transition From Asymptomatic Colonization to Disease. mBio 4 (4), e00438-13. doi: 10.1128/mBio.00438-13

Mclean, K. T., Tikhomirova, A., Brazel, E. B., Legendre, S., Haasbroek, G., Minhas, V., et al. (2020). Site-Specific Mutations of GalR Affect Galactose Metabolism in Streptococcus Pneumoniae. J. Bacteriol. 203 (1), e00180-20. doi: 10.1128/ JB.00180-20

Mills, N., Best, E. J., Murdoch, D., Souter, M., Neeff, M., Anderson, T., et al. (2015). What is Behind the Ear Drum? The Microbiology of Otitis Media and the Nasopharyngeal Flora in Children in the Era of Pneumococcal Vaccination. J. Paediatr. Child Health 5 (3), 300-306. doi: 10.1111/jpc.12710

Minhas, V., Paton, J. C., and Trappetti, C. (2021). Sickly Sweet - How Sugar Utilization Impacts Pneumococcal Disease Progression. Trends Microbiol. 29 (9), 768-771. doi: 10.1016/j.tim.2021.01.016

Mokrzan, E. M., Johnson, T. J., and Bakaletz, L. O. (2019). Expression of the Nontypeable Haemophilus Influenzae Type IV Pilus Is Stimulated by Coculture With Host Respiratory Tract Epithelial Cells. Infect. Immun. 87 (12), e0070419. doi: 10.1128/IAI.00704-19

Mokrzan, E. M., Novotny, L. A., Brockman, K. L., and Bakaletz, L. O. (2018). Antibodies Against the Majority Subunit (PilA) of the Type IV Pilus of Nontypeable Haemophilus Influenzae Disperse Moraxella Catarrhalis From a Dual-Species Biofilm. mBio 9 (6), e02423-e02418. doi: 10.1128/mBio.02423-18 
Monasta, L., Ronfani, L., Marchetti, F., Montico, M., Vecchi Brumatti, L., Bavcar, A., et al. (2012). Burden of Disease Caused by Otitis Media: Systematic Review and Global Estimates. PloS One 7 (4), e36226. doi: 10.1371/journal.pone.0036226

Motib, A. S., Al-Bayati, F., Manzoor, I., Shafeeq, S., Kadam, A., Kuipers, O. P., et al. (2019). TprA/PhrA Quorum Sensing System Has a Major Effect on Pneumococcal Survival in Respiratory Tract and Blood, and Its Activity Is Controlled by CcpA and GlnR. Front. Cell Infect. Microbiol. 9. doi: 10.3389/ fcimb.2019.00326

Motib, A., Guerreiro, A., Al-Bayati, F., Piletska, E., Manzoor, I., Shafeeq, S., et al. (2017). Modulation of Quorum Sensing in a Gram-Positive Pathogen by Linear Molecularly Imprinted Polymers With Anti-Infective Properties. Angew Chem. Int. Ed Engl. 56 (52), 16555-16558. doi: 10.1002/anie.201709313

Murphy, T. F., Brauer, A. L., Pettigrew, M. M., Lafontaine, E. R., and Tettelin, H. (2019). Persistence of Moraxella Catarrhalis in Chronic Obstructive Pulmonary Disease and Regulation of the Hag/MID Adhesin. J. Infect. Dis. 219 (9), 1448-1455. doi: 10.1093/infdis/jiy680

Paixao, L., Caldas, J., Kloosterman, T. G., Kuipers, O. P., Vinga, S., and Neves, A. R. (2015a). Transcriptional and Metabolic Effects of Glucose on Streptococcus Pneumoniae Sugar Metabolism. Front. Microbiol. 6, 1041. doi: 10.3389/ fmicb.2015.01041

Paixao, L., Oliveira, J., Verissimo, A., Vinga, S., Lourenco, E. C., Ventura, M. R., et al. (2015b). Host Glycan Sugar-Specific Pathways in Streptococcus Pneumoniae: Galactose as a Key Sugar in Colonisation and Infection [Corrected]. PloS One 10 (3), e0121042. doi: 10.1371/journal.pone.0121042

Parrish, J. M., Soni, M., and Mittal, R. (2019). Subversion of Host Immune Responses by Otopathogens During Otitis Media. J. Leukocyte Biol. 106 (4), 943-956. doi: 10.1002/JLB.4RU0119-003R

Paton, J. C., and Trappetti, C. (2019). Stand by to Repel Boarders. Nat. Microbiol. 4 (1), 8-9. doi: 10.1038/s41564-018-0324-2

Pettigrew, M. M., Ahearn, C. P., Gent, J. F., Kong, Y., Gallo, M. C., Munro, J. B., et al. (2018). Haemophilus Influenzae Genome Evolution During Persistence in the Human Airways in Chronic Obstructive Pulmonary Disease. Proc. Natl. Acad. Sci. U. S. A. 115 (14), E3256-E3265. doi: 10.1073/pnas.1719654115

Pettigrew, M. M., Marks, L. R., Kong, Y., Gent, J. F., Roche-Hakansson, H., and Hakansson, A. P. (2014). Dynamic Changes in the Streptococcus Pneumoniae Transcriptome During Transition From Biofilm Formation to Invasive Disease Upon Influenza A Virus Infection. Infect. Immun. 82 (11), 4607-4619. doi: 10.1128/IAI.02225-14

Phillips, Z. N., Husna, A. U., Jennings, M. P., Seib, K. L., and Atack, J. M. (2019). Phasevarions of Bacterial Pathogens - Phase-Variable Epigenetic Regulators Evolving From Restriction-Modification Systems. Microbiol. (Reading). 165 (9), 917-928. doi: 10.1099/mic.0.000805

Poole, J., Foster, E., Chaloner, K., Hunt, J., Jennings, M. P., Bair, T., et al. (2013). Analysis of Nontypeable Haemophilus Influenzae Phase-Variable Genes During Experimental Human Nasopharyngeal Colonization. J. Infect. Dis. 208 (5), 720-727. doi: 10.1093/infdis/jit240

Riesbeck, K. (2020). Complement Evasion by the Human Respiratory Tract Pathogens Haemophilus Influenzae and Moraxella Catarrhalis. FEBS Lett. 594 (16), 2586-2597. doi: 10.1002/1873-3468.13758

Riesbeck, K., and Nordstrom, T. (2006). Structure and Immunological Action of the Human Pathogen Moraxella Catarrhalis IgD-Binding Protein. Crit. Rev. Immunol. 26 (4), 353-376. doi: 10.1615/critrevimmunol.v26.i4.40

Robledo-Avila, F. H., Ruiz-Rosado, J. D., Partida-Sanchez, S., and Brockman, K. L. (2020). A Bacterial Epigenetic Switch in Non-Typeable Haemophilus Influenzae Modifies Host Immune Response During Otitis Media. Front. Cell Infect. Microbiol. 10, 512743. doi: 10.3389/fcimb.2020.512743

Santana, E. A., Harrison, A., Zhang, X., Baker, B. D., Kelly, B. J., White, P., et al. (2014). HrrF is the Fur-Regulated Small RNA in Nontypeable Haemophilus influenzae. PloS One 9 (8), e105644. doi: 10.1371/journal.pone.0105644

Schaar, V., Paulsson, M., Morgelin, M., and Riesbeck, K. (2013). Outer Membrane Vesicles Shield Moraxella Catarrhalis Beta-Lactamase From Neutralization by Serum IgG. J. Antimicrob. Chemother. 68 (3), 593-600. doi: 10.1093/jac/dks444

Schilder, A. G. M., Chonmaitree, T., Cripps, A. W., Rosenfeld, R. M., Casselbrant, M. L., Haggard, M. P., et al. (2016). Otitis Media. Nat. Rev. Dis. Primers. 2 (1), 16063. doi: $10.1038 / \mathrm{nrdp} .2016 .63$

Singh, B., Alvarado-Kristensson, M., Johansson, M., Hallgren, O., WestergrenThorsson, G., Morgelin, M., et al. (2016). The Respiratory Pathogen Moraxella
Catarrhalis Targets Collagen for Maximal Adherence to Host Tissues. mBio 7 (2), e00066. doi: 10.1128/mBio.00066-16

Slager, J., Kjos, M., Attaiech, L., and Veening, J. W. (2014). Antibiotic-Induced Replication Stress Triggers Bacterial Competence by Increasing Gene Dosage Near the Origin. Cell 157 (2), 395-406. doi: 10.1016/j.cell.2014.01.068

Spaniol, V., Bernhard, S., and Aebi, C. (2015). Moraxella Catarrhalis AcrABOprM Efflux Pump Contributes to Antimicrobial Resistance and is Enhanced During Cold Shock Response. Antimicrob. Agents Chemother. 59 (4), 18861894. doi: 10.1128/AAC.03727-14

Spaniol, V., Troller, R., Schaller, A., and Aebi, C. (2011). Physiologic Cold Shock of Moraxella Catarrhalis Affects the Expression of Genes Involved in the Iron Acquisition, Serum Resistance and Immune Evasion. BMC Microbiol. 11, 182. doi: 10.1186/1471-2180-11-182

Spaniol, V., Wyder, S., and Aebi, C. (2013). RNA-Seq-Based Analysis of the Physiologic Cold Shock-Induced Changes in Moraxella Catarrhalis Gene Expression. PloS One 8 (7), e68298. doi: 10.1371/journal.pone.0068298

Srikhanta, Y. N., Maguire, T. L., Stacey, K. J., Grimmond, S. M., and Jennings, M. P. (2005). The Phasevarion: A Genetic System Controlling Coordinated, Random Switching of Expression of Multiple Genes. Proc. Natl. Acad. Sci. U. S. A. 102 (15), 5547-5551. doi: 10.1073/pnas.0501169102

Steele, K. H., O'connor, L. H., Burpo, N., Kohler, K., and Johnston, J. W. (2012). Characterization of a Ferrous Iron-Responsive Two-Component System in Nontypeable Haemophilus influenzae. J. Bacteriol. 194 (22), 6162-6173. doi: 10.1128/JB.01465-12

Su, Y. C., Jalalvand, F., Thegerström, J., and Riesbeck, K. (2018). The Interplay Between Immune Response and Bacterial Infection in COPD: Focus Upon Non-Typeable Haemophilus influenzae. Front. Immunol. 9, 2530. doi: 10.3389/ fimmu.2018.02530

Szelestey, B. R., Heimlich, D. R., Raffel, F. K., Justice, S. S., and Mason, K. M. (2013). Haemophilus Responses to Nutritional Immunity: Epigenetic and Morphological Contribution to Biofilm Architecture, Invasion, Persistence and Disease Severity. PloS Pathogens. 9 (10), e1003709. doi: 10.1371/ journal.ppat.1003709

Tan, A., Blakeway, L. V., Taha, Yang, Y., Zhou, Y., Atack, J. M., et al. (2020). Moraxella Catarrhalis Phase-Variable Loci Show Differences in Expression During Conditions Relevant to Disease. PloS One 15 (6), e0234306. doi: 10.1371/journal.pone.0234306

Thornton, R. B., Rigby, P. J., Wiertsema, S. P., Filion, P., Langlands, J., Coates, H. L., et al. (2011). Multi-Species Bacterial Biofilm and Intracellular Infection in Otitis Media. BMC Pediatr. 11, 94. doi: 10.1186/1471-2431-11-94

Trappetti, C., Potter, A. J., Paton, A. W., Oggioni, M. R., and Paton, J. C. (2011). LuxS Mediates Iron-Dependent Biofilm Formation, Competence, and Fratricide in Streptococcus Pneumoniae. Infect. Immun. 79 (11), 4550-4558. doi: 10.1128/IAI.05644-11

Unal, C. M., Singh, B., Fleury, C., Singh, K., Chavez De Paz, L., Svensater, G., et al. (2012). QseC Controls Biofilm Formation of Non-Typeable Haemophilus Influenzae in Addition to an AI-2-Dependent Mechanism. Int. J. Med. Microbiol. 302 (6), 261-269. doi: 10.1016/j.ijmm.2012.07.013

Valente, C., Dawid, S., Pinto, F. R., Hinds, J., Simoes, A. S., Gould, K. A., et al. (2016). The Blp Locus of Streptococcus Pneumoniae Plays a Limited Role in the Selection of Strains That Can Cocolonize the Human Nasopharynx. Appl. Environ. Microbiol. 82 (17), 5206-5215. doi: 10.1128/AEM.01048-16

Van Hoecke, H., De Paepe, A. S., Lambert, E., Van Belleghem, J. D., Cools, P., Van Simaey, L., et al. (2016). Haemophilus Influenzae Biofilm Formation in Chronic Otitis Media With Effusion. Eur. Arch. Otorhinolaryngol. 273 (11), 3553-3560. doi: 10.1007/s00405-016-3958-9

Verhaegh, S. J. C., Snippe, M. L., Levy, F., Verbrugh, H. A., Jaddoe, V. W. V., Hofman, A., et al. (2011). Colonization of Healthy Children by Moraxella Catarrhalis is Characterized by Genotype Heterogeneity, Virulence Gene Diversity and Co-Colonization With Haemophilus influenzae. Microbiol. (Reading). 1571 (Pt 1), 169-178. doi: 10.1099/mic.0.042929-0

Wang, W., Joslin, S. N., Pybus, C., Evans, A. S., Lichaa, F., Brautigam, C. A., et al. (2014). Identification of an Outer Membrane Lipoprotein Involved in Nasopharyngeal Colonization by Moraxella Catarrhalis in an Animal Model. Infect. Immun. 82 (6), 2287-2299. doi: 10.1128/IAI.01745-14

Wang, C. Y., Patel, N., Wholey, W. Y., and Dawid, S. (2018). ABC Transporter Content Diversity in Streptococcus Pneumoniae Impacts Competence 
Regulation and Bacteriocin Production. Proc. Natl. Acad. Sci. U. S. A. 115 (25), E5776-E5785. doi: 10.1073/pnas.1804668115

Weiser, J. N., Ferreira, D. M., and Paton, J. C. (2018). Streptococcus Pneumoniae: Transmission, Colonization and Invasion. Nat. Rev. Microbiol. 16 (6), 355-367. doi: 10.1038/s41579-018-0001-8

Weyder, M., Prudhomme, M., Berge, M., Polard, P., and Fichant, G. (2018). Dynamic Modeling of Streptococcus Pneumoniae Competence Provides Regulatory Mechanistic Insights Into Its Tight Temporal Regulation. Front. Microbiol. 9. doi: 10.3389/fmicb.2018.01637

Whitby, P. W., Morton, D. J., Vanwagoner, T. M., Seale, T. W., Cole, B. K., Mussa, H. J., et al. (2012). Haemophilus Influenzae OxyR: Characterization of its Regulation, Regulon and Role in Fitness. PloS One 7 (11), e50588. doi: 10.1371/ journal.pone.0050588

Whitby, P. W., Vanwagoner, T. M., Seale, T. W., Morton, D. J., and Stull, T. L. (2013). Comparison of Transcription of the Haemophilus Influenzae Iron/ Heme Modulon Genes In Vitro and In Vivo in the Chinchilla Middle Ear. BMC Genomics 14 (1), 925. doi: 10.1186/1471-2164-14-925

Whittaker, R., Economopoulou, A., Dias, J. G., Bancroft, E., Ramliden, M., Celentano, L. P., et al. (2017). Epidemiology of Invasive Haemophilus Influenzae Disease, Europe 2007-2014. Emerg. Infect. Dis. 23 (3), 396-404. doi: 10.3201/eid2303.161552

Wholey, W. Y., Kochan, T. J., Storck, D. N., and Dawid, S. (2016). Coordinated Bacteriocin Expression and Competence in Streptococcus Pneumoniae Contributes to Genetic Adaptation Through Neighbor Predation. PloS Pathog. 12 (2), e1005413. doi: 10.1371/journal.ppat.1005413

Yadav, M. K., Go, Y. Y., Chae, S. W., Park, M. K., and Song, J. J. (2020). Asian Sand Dust Particles Increased Pneumococcal Biofilm Formation In Vitro and Colonization in Human Middle Ear Epithelial Cells and Rat Middle Ear Mucosa. Front. Genet. 11. doi: 10.3389/fgene.2020.00323
Yadav, M. K., Vidal, J. E., Go, Y. Y., Kim, S. H., Chae, S. W., and Song, J. J. (2018). The LuxS/AI-2 Quorum-Sensing System of Streptococcus Pneumoniae Is Required to Cause Disease, and to Regulate Virulence- and MetabolismRelated Genes in a Rat Model of Middle Ear Infection. Front. Cell Infect. Microbiol. 8. doi: $10.3389 /$ fcimb.2018.00138

Zhi, X., Abdullah, I. T., Gazioglu, O., Manzoor, I., Shafeeq, S., Kuipers, O. P., et al. (2018). Rgg-Shp Regulators Are Important for Pneumococcal Colonization and Invasion Through Their Effect on Mannose Utilization and Capsule Synthesis. Sci. Rep. 8 (1), 6369. doi: 10.1038/s41598-018-24910-1

Conflict of Interest: The authors do not have any commercial interests. KR is participating in projects supported by Pfizer and has been collaborating with GSK.

The remaining authors declare that the research was conducted in the absence of any commercial or financial relationships that could be construed as a potential conflict of interest.

Publisher's Note: All claims expressed in this article are solely those of the authors and do not necessarily represent those of their affiliated organizations, or those of the publisher, the editors and the reviewers. Any product that may be evaluated in this article, or claim that may be made by its manufacturer, is not guaranteed or endorsed by the publisher.

Copyright (๑) 2022 Janoušková, Straw, Su and Riesbeck. This is an open-access article distributed under the terms of the Creative Commons Attribution License (CC BY). The use, distribution or reproduction in other forums is permitted, provided the original author(s) and the copyright owner(s) are credited and that the original publication in this journal is cited, in accordance with accepted academic practice. No use, distribution or reproduction is permitted which does not comply with these terms. 